\title{
Guidelines for reproducing blast exposures in the laboratory
}

\author{
Tyson Josey, ${ }_{1}^{1}$ S Ouellet, ${ }^{2}$ D Bieler, ${ }^{3}$ I Cernak, ${ }^{4}$ A Franke, ${ }^{3}$ R Gupta, ${ }^{5}$ E Kirkman, ${ }^{6}$ \\ M J Leggieri Jr, ${ }^{5} \mathrm{H}$ Orru, $^{7}$ M Philippens, ${ }^{8}$ M Risling, ${ }^{9} \mathrm{~J}$ C Sarron, ${ }^{10}$ S Skriudalen, ${ }^{11}$ \\ J A Teland, ${ }^{11}$ S Watts, ${ }^{6}$ S Bjarnason ${ }^{1}$
}

${ }^{1}$ Suffield Research Centre,

Defence Research and

Development Canada, Medicine

Hat, Alberta, Canada

${ }^{2}$ Valcartier Research Centre,

Defence Research and

Development Canada, Quebec

City, Quebec, Canada

${ }^{3}$ Department of Trauma

Surgery and Orthopaedics,

Reconstructive Surgery, Hand

Surgery and Burn Medicine,

German Armed Forces Central

Hospital of Koblenz, Koblenz,

Germany

${ }^{4}$ STARR-C, LLC, Edmonton, Alberta, Canada

${ }^{5}$ U.S. Army Medical Research and Materiel Command, DoD

Blast Injury Research Program

Executive Agency, Maryland,

Maryland, USA

${ }^{6}$ CBR Division, Wiltshire, UK Institute of Family Medicine and Public Health, University of Tartu, Tartu, Tartu, Estonia ${ }^{8}$ TNO Rijswijk, Rijswijk, The Netherlands

${ }^{9}$ Department of Neuroscience, Karolinska Institute, Stockholm, UK

${ }^{10}$ Direction centrale du service de santé des armées DCSSA

Sous-direction "Plans -

Capacités", Paris, France

${ }^{11}$ Protection Division, Norwegian Defence Research Establishment (FFI), Kjeller, Norway

\section{Correspondence to}

Tyson Josey, Suffield Research Centre, Defence Research and Development Canada, Medicine Hat, Alberta T1A 8K6, Canada; tyson.josey@drdc-rddc.gc.ca

Received 20 March 2018 Accepted 22 March 2018 Published Online First 7 May 2018

Check for updates

To cite: Josey T, Ouellet $\mathrm{S}$,

Bieler $\mathrm{D}$, et al.

I R Army Med Corps

2019:165:10-14.

\section{INTRODUCTION}

Discussions at a NATO Health Factors and Medicine Symposium 207 (HFM-SYM-207) revealed the importance of a systematic approach to understanding blast injuries much like the well-established approach used to solve the classical toxicology problem where the aetiology of the injury requires an understanding of the dose, mechanism of delivery of the dosage and dose-response endpoints. ${ }^{1}$ To address the above recommendation and to develop a specific NATO activity devoted to the toxicology of blast exposure, a proposal titled 'Environmental Toxicology of Blast Exposures: Injury Metrics, Modeling, Methods and Standards' was approved which resulted in the establishment of a NATO HFM Research Task Group (RTG; HFM-234 (RTG)) with the following deliverables.

The guidelines developed under HFM-234 are intended to provide blast injury research laboratories with a fundamental set of characteristics that need to be collected and described when generating blast pressure waves. It is not the intention to prescribe how to create the blast pressure waves but to provide an awareness of what needs to be taken into account, measured and updated when creating blast exposures; the objectives of this document are to

(i) Raise awareness regarding the complexities and pitfalls of blast injury research.

(ii) Standardise and promote good practices.

(iii) Help the community to generate valid and comparable results.

(iv) Increase the quality of publications in this field of research.

\section{BACKGROUND}

These guidelines were developed in response to the considerable variability and reporting of methodologies used to create blast exposure. This has resulted in an inability to compare results and conclusions generated by different research institutes engaged in blast injury research. Significant variability exists in the methodologies used by different groups to create the blast pressure wave and often there is a lack of information reported in publications, preventing a complete understanding of the experimental conditions. A significant portion of blast injury research is focused on neurotrauma and the mild traumatic brain injury (mTBI) research community is multidisciplinary in nature with a good portion of the community not necessarily having expertise in blast physics/engineering. Not fully understanding and characterising the exposure metrics has resulted in a detrimental effect on developing evidence-based solutions for problems such as blast-induced mTBI.

\section{REPRODUCING BLAST EXPOSURE AND CONDUCTING BLAST EXPERIMENTS}

It is imperative that there is an understanding of actual operational blast exposures in order to simulate such exposures in the laboratory especially for reproducing clinically relevant injury patterns.

A common device in many institutions is a shock tube used to simulate free-field blast, which in its simplest form is represented by an instantaneous rise in pressure and an exponential decay followed by a negative phase. $^{2}$ Field testing requires the use of an open area and an explosive charge to generate the pressure wave. Each method of generating pressure waves has advantages and limitations. The consequences of not understanding the limitations and physics/engineering of the different methods have the potential to cause significant errors in interpretation of measured endpoints. In a classical toxicological sense, if the 'dose' is not well characterised then it is difficult to properly understand the 'response' of the target/test system.

\section{SHOCK/BLAST TUBES}

Shock tubes have quick turnaround times and can improve repeatability over free-field blast experiments. The range of achievable wave profiles and positive phase durations are dependent on the design of the shock tube. It is recommended that a particular threat be identified and the shock tube be designed to the threat.

A compressed gas shock tube is essentially a tube with two sections (driver and driven) separated by a frangible diaphragm. The driver section is charged with a high-pressure gas and, when the diaphragm is ruptured, this high-pressure gas rushes into the driven section, developing a shock wave (figure 1).

A basic shock tube will not accurately reproduce the flow conditions and wave dynamics generated from a free-field explosive. An enhanced shock tube is better suited for the purpose of investigating blast injury. This type of shock tube resolves a number of issues seen in conventional shock tubes and is tailored to produce blast waves. The advanced blast simulator (ABS) used at Defence Research and Development Canada-Suffield Research Centre has four sections: the driver, expansion/transition section, driven section and end wave eliminator 

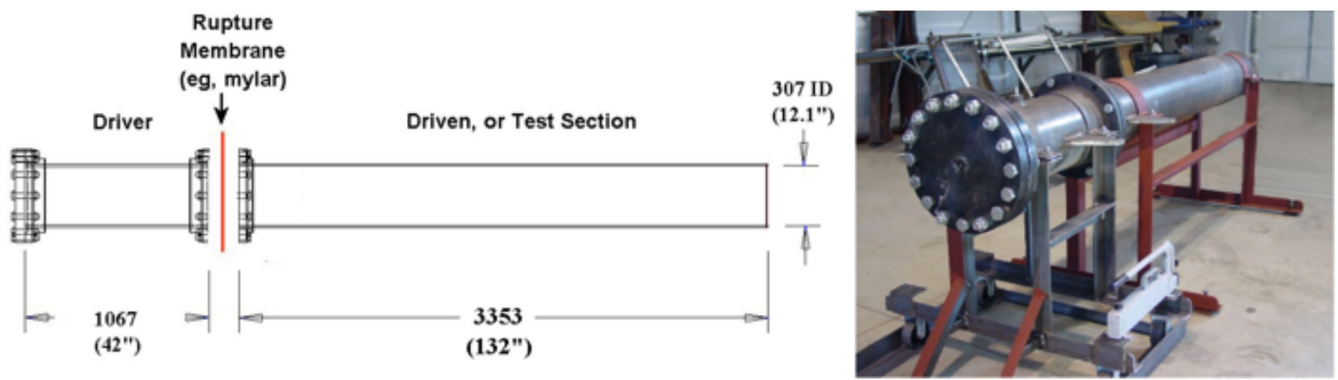

Figure 1 Schematic of a gas-driven shock tube.

(EWE). The driver is made in such a way that the driver gas is allowed to expand and is then tailored by the transition section (figure 2). Low-molecular-weight driver gases produce higher peak pressures, shorter durations and a much less prominent negative phase (figure 3). The target is placed inside of the driven section, at a location where the flow is fully developed. When animal testing is used, the driver gas should be restricted from reaching the test subject so as not to complicate the insult experienced by the test subject. ${ }^{3}$

The ABS uses a controlled venting device or End Wave Eliminator (EWE) that is tuned for each blast condition and allows for the elimination of rarefaction waves and secondary waves travelling back up the tube into the test section (figures 4 and 5).

There are a number of potential issues when using shock tubes to investigate mTBI. One way to enhance repeatability is by obtaining a repeatable diaphragm burst behaviour and consistent driver pressure. The type of diaphragm failure can be a petalling-type failure or a fragmentation rupture of the diaphragm. With petalling failure, the orifice created needs to prevent a jet from forming. Fragments need to be controlled so they do not cause issues by impacting the test subjects.

Typical static pressure measurements do not provide the necessary insights to understand the loading on the subject and the reported peak is often an artefact of the gauge. Understanding the flow field requires knowing the density, velocity and pressure of the blast wave. To simulate a blast wave, the flow field must match the free-field conditions. Matching the static pressure provides only partial insight. The use of a Pitot tube in the direction of the flow provides the resultant total pressure and the dynamic pressure can be calculated (figure 6). The dynamic pressure cannot be ignored as it may be a source of significant loading, especially if the test subject is positioned outside the blast tube. Test subjects placed outside are subject to severe loads and wave perturbations that are not observed in a free-field blast environment. The diameter of the shock tube will limit the size and geometry of the test subject that can be used inside the tube. A rule of thumb is that the test subject be limited to block no more than $10 \%$ of the cross-sectional area. Along with numerical models, measurements in the centre of the tube should be conducted to characterise the boundary layer effect and to determine the planarity of the wave.

\section{FIELD TESTING}

Field testing (figure 7) is often seen as the closest representation of an operational blast scenario. However, conducting a full-scale field blast trial does not guarantee that loading conditions are repeatable and suitable. High repeatability can be achieved when experiments are carefully executed and when appropriate equipment is used. The inherent source of variation in field experiments often comes from the explosive charge. In order to increase repeatability, great care needs to be taken during the preparation of the charge. Using explosives from a similar production batch, conducting precise weighing and ensuring adherence to geometry will reduce variability in the formation of the blast wave on detonation of the charge. The set-up for positioning the charge should also be well thought-out and highly controlled.

It is often incorrectly assumed that targets directly aligned with the blast flow will be exposed to a relatively unperturbed blast wave. It is critical to understand and control the effects that surrounding obstacles, reflecting surfaces, sensors mounts and other experimental set-ups can have on the blast wave flow field. These reflections should be carefully identified, characterised and preferably eliminated. High-speed videography can help identify sources of reflections, but in general, the best practice is to understand and plan the test layout carefully to avoid them.

The near-field regime is typically defined within the radius of the fireball. Within that range, the medium is multiphase (mix of air and detonation products), the shock structure is likely very complex and is not the ideal regime to conduct controlled blast testing. The midfield regime (1-10 radii of the fireball) is where most full-scale target field tests are conducted. It is practical in terms of required size for the test site, it is operationally relevant and the blast dose can be adjusted to be in the range of potential injury thresholds. However, potential interaction of the target with the fireball and wave uniformity, structure, propagation direction and curvature all need to be monitored as they can vary substantially depending on stand-off distance and charge height of burst (figure 8 ). In the far-field (over 10 radii of the fireball)

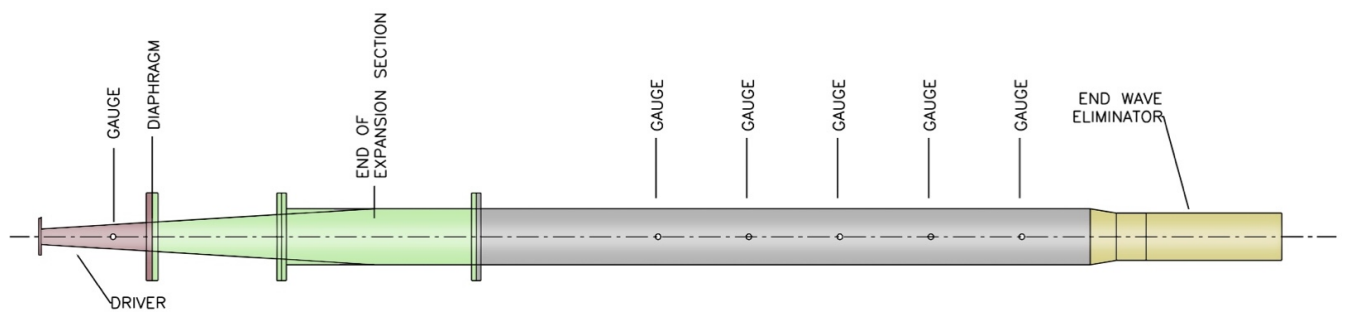

Figure 2 Schematic representation of an advanced blast simulator. 


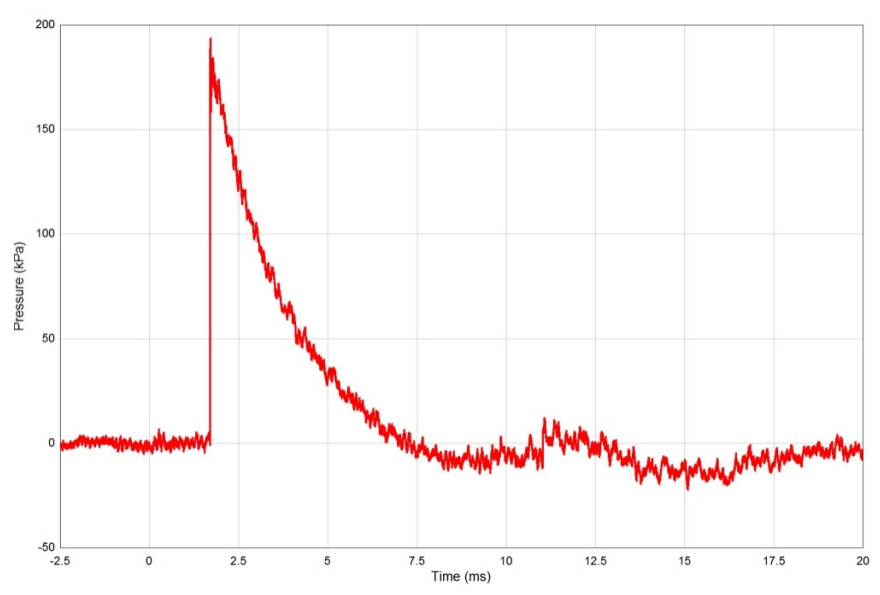

Figure 3 Example of achievable wave profile with an advanced blast simulator.

regime, the shock is more likely to be uniform and curvature is low and at the scale of the biological target. This is the ideal regime to maximise repeatability but unfortunately it is often impractical and the resulting exposure condition may be too low for subtle injury studies.

In open free-field testing, the blast wave can be monitored by deploying an array of static pressure gauges pointing towards the blast source. If other targets are used, they should have an associated reference pressure gauge. Shock velocity derived from two successive pressure gauges, along with local air properties, will allow calculation of other quantities of interest such as dynamic and reflected pressure. Pitot probes can also be used to measure the total pressure history and derive the dynamic pressure history under certain conditions.

The propagation direction of the blast wave relative to the target dictates the manner in which the blast wave reflects off and diffracts around the target. As such, it affects the evolution of the transient loading condition experienced by the target; it should be selected carefully to represent the desired scenario. Target support needs to be considered as it will affect the target response (eg, head models using a rigid neck may not capture an important aspect of the target response). The presence of reflecting surfaces (walls and ground) will result in reflected waves with different propagation directions. In such conditions, numerical simulation can be very useful to characterise the blast field around a potential target and evaluate the intensity and propagation direction of the incident and reflected blast waves.

A more rigorous way of monitoring the exposure conditions of a target is to monitor the loading directly on the target. Typical sensors that can be used include accelerometers, pressure transducers (internal and external), strain gauges and force

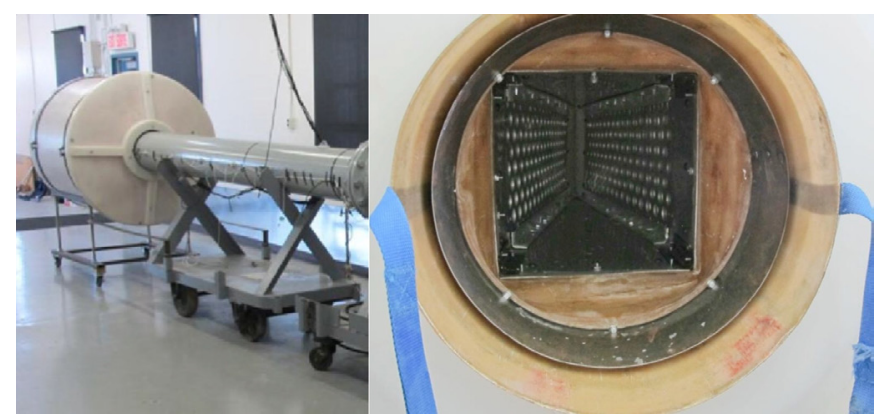

Figure 4 Example of an end wave eliminator.

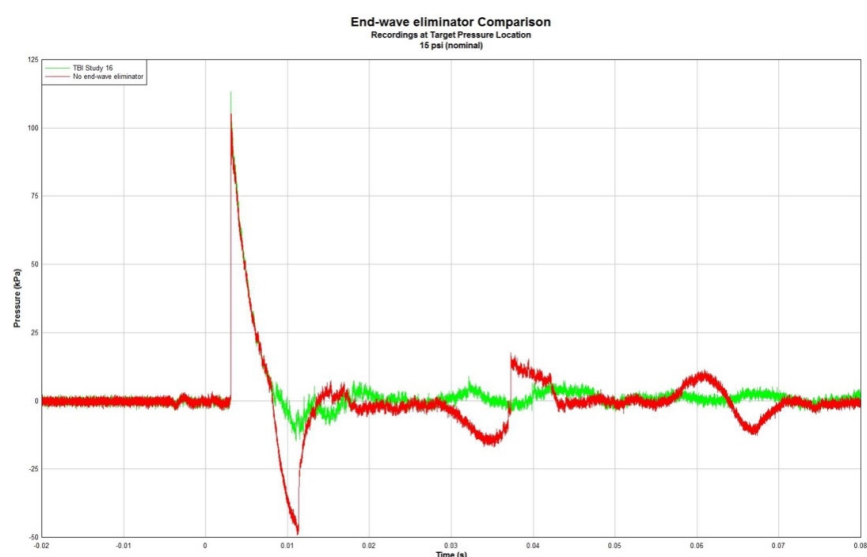

Figure 5 Example traces without (red) and with (green) end wave eliminator.

transducers. The potential influence of the sensor on the response of the target should be evaluated. Sensors should not restrain or modify the local or global motion of the target. In general, size of the sensors should be minimised as much as possible and be an order of magnitude smaller than the target itself. Using sensors with animal models, postmortem human subjects (PMHS) or physical models often means mounting sensors on/in deformable surfaces or structures. Great care must be taken to make sure that there is intimate contact between the sensor and the surface/structure of interest, and that it remains unchanged during the loading and unloading. High-speed imagery can be useful to track target motion. Target deformation can be evaluated through the use of Digital Image Correlation techniques. High-speed imagery equipment needs to be protected adequately and positioned at a reasonable distance from the explosion. The shock wave, fireball, air flow and detonation products can all distort or obstruct the field of view.

To accurately reproduce blast exposure in a laboratory environment implies that the instrumentation used to measure the blast exposure is also accurate and adequate. Sensor bandwidth is particularly important when measuring initial peak values generated by the passage of a shock because the upper bound of the bandwidth (highest frequency) puts the limit on the shortest resolvable signal rise time. If the sensor cannot respond fast enough to capture the initial signal rise, initial peak values will be underestimated. As a general rule, sensor bandwidth should be maximised whenever possible. The cut-off frequencies

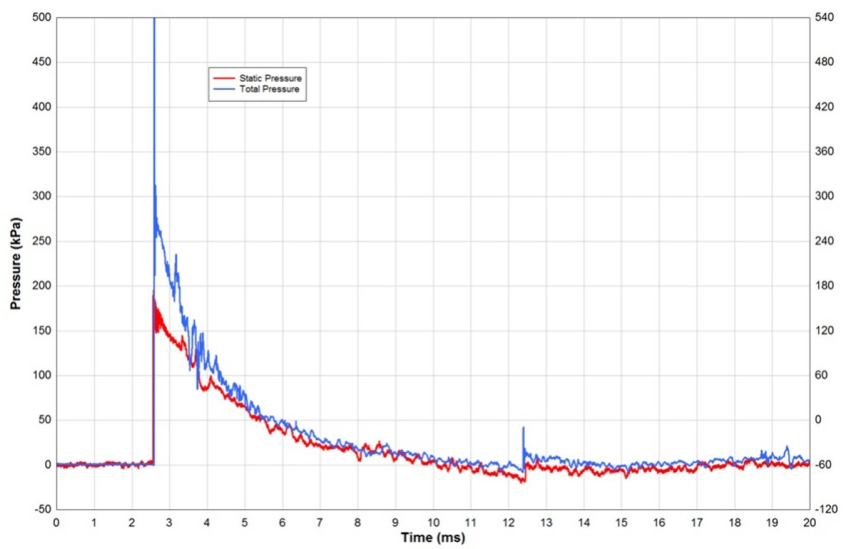

Figure 6 Characterisation of the exposure using a wall-mounted incident pressure gauge and a Pitot probe to measure total pressure. 


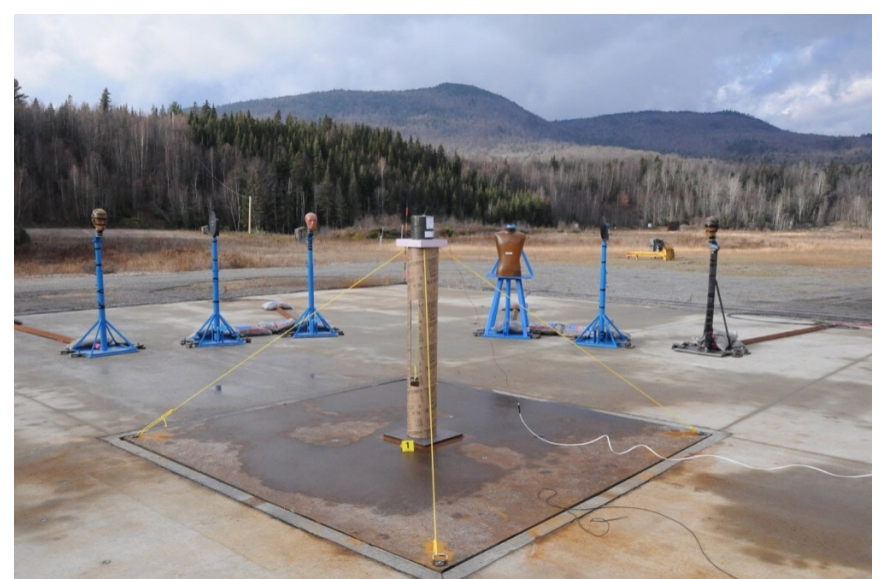

Figure 7 A example of full-scale explosive test set-up simulating the above-ground detonation of a large charge, centrally located relative to a series of targets.

of embedded mechanical or electrical filters should not be in the relevant frequency range of the measurement. The Nyquist theorem stipulates that the sampling frequency should be at least two times the highest expected frequency in the perturbation being measured. However, to avoid the need for signal reconstruction, the common practice is to use a sampling frequency 5-10 times the highest expected frequency in the signal. The use of an anti-aliasing filter is recommended since other outside stimuli containing even higher frequencies may be undersampled and superposed on the signal of interest

\section{REPORTING RESEARCH WORK}

The information that must be addressed and then reported has been broken down into four constituent parts: research rationale, blast characteristics, target exposure characteristics and target response. A clear articulation of the rationale for the research must be provided and must include, but is not limited to, the following:

i. Aim of the experiment.

ii. Hypothesis to be tested.

iii. How the experiment answers the hypothesis.

iv. How the experiment relates to real-world operational conditions.

v. Why the exposure level was chosen.

A distinction needs to be made between blast characteristics and exposure/target loading conditions. The blast

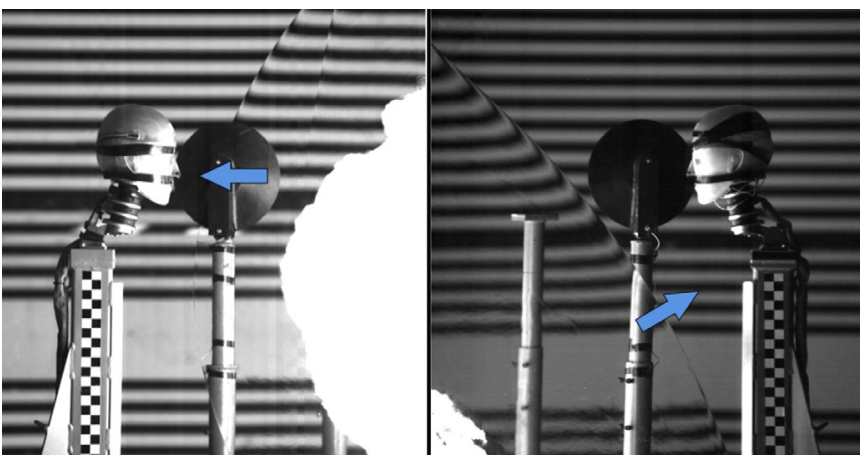

Figure 8 An example of varying exposure conditions in the midfield regime. Both images are from tests with a $5 \mathrm{~kg}$ explosive charge detonated at the same stand-off distance. On the left side, the height of burst was $1.5 \mathrm{~m}$. On the right, the height of burst was $0.2 \mathrm{~m}$. The propagation direction is shown by the blue arrows. characteristics define a scenario independent of any target. The information that should be reported includes

i. Blast simulation method.

ii. Ambient conditions.

iii. Data acquisition and processing.

iv. Blast characteristics at target location.

v. Duration of recording.

vi. Level of reproducibility and number of repeats.

vii. Effects of sample holders and other structures.

viii. Exposure level along the propagation direction.

The target exposure, or loading conditions, is the result of the interaction between the blast and the target. It is influenced by various factors including target type, geometry, position, orientation, mounting and protection. The emphasis is put on the reporting of a set of parameters which fully describe the loading conditions.

The researcher should provide a description of the type of target (eg, physical surrogate, animal model, PMHS, cellular material, other object) and the selection rationale for the study along with discussing advantages and disadvantages of the target. If novel targets are used, provide information to substantiate the choice of targets. Accurate, detailed information with respect to the target positioning and orientation should be specified (eg, free-field test: distance from the centre of the charge, as well as height of the specimen and orientation in the three axes; shock tubes: the distance from the driver to the specimen and orientation).

Protective equipment (PE) when used with the target needs to be described and any modifications reported. A detailed description of how the system was fitted and secured on the target is necessary. Any interaction of the PE with the target needs to be analysed as it may play a critical role in the response. For response monitoring with sensors, the relevant sensor specifications should be provided along with the location, mounting and signal sampling/processing. History over the full duration of the experiment should be provided. Surface pressure data add to the interpretation of the target response and provide a true measurement of direct loading on the target. Strain data provide a direct local measurement of the effect of the loading on the target structure. They can provide a global understanding of the modes of deformation of the target. When available, data from multiple sensors should be analysed jointly to identify the origin and propagation direction of stress waves. If animal models are used as a target, the rationale, physiological parameters measured pre-exposure and postexposure should be collected (eg, telemetry devices) and reported. The mounting or holding system needs to be described and its effects need to be taken into account. For more detail on mounting animal/live tissue targets, see NATO HFM 'Guidelines for Using Animal Models in Blast Injury Research’, 2016, Paris, France.

\section{CONCLUSIONS}

Creating blast exposures in a laboratory setting is not a trivial undertaking; it requires expertise, knowledge and capabilities in blast wave physics, as well as engineering. Understanding the complexities of generating reproducible blast waves is critical for recreating relevant military exposures in the laboratory. As with other toxicology research, characterising the 'dose' is crucial to understanding the 'response' of a test system. Whether shock/blast tubes are used or free-field blast trials are performed, several sources of interference must be recognised, and either controlled or explained as to the effect 
on the test system, and the resulting limitations to the results generated.

Given the complexity of blast exposure, understanding the injury effects that may result from the different components of a blast (eg, pressure, duration) is sometimes needed before the overall effect from a blast exposure can be explained, depending on the research question asked. This is similar to understanding the toxicology of complex mixtures of chemicals where the toxic effects of the individual components are required before understanding the effects from the mixture. Given the response is in a complex biological system, understanding 'mixture' effects is not easy. Combining complex exposures such as blast with complex responders such as humans makes the interpretation even more challenging.

These guidelines are the outcome of an attempt to understand how blast exposures can be created and the information that is required to allow for experimental work from different laboratories/institutions to be compared. Ultimately this will advance the state of the science and result in the best evidence possible to inform those responsible for the protection and care of military members.

Funding The authors have not declared a specific grant for this research from any funding agency in the public, commercial or not-for-profit sectors.

Competing interests None declared.

Patient consent Not required.

Provenance and peer review Not commissioned; internally peer reviewed.

(c) Article author(s) (or their employer(s) unless otherwise stated in the text of the article) 2019. All rights reserved. No commercial use is permitted unless otherwise expressly granted.

\section{REFERENCES}

1 NATO HFM-234 (RTG). "Environmental Toxicology of Blast Exposures: Injury Metrics, Modeling, Methods and Standards" Kick-Off Meeting and Program of Work. Paris, France: NATO HFM-234 (RTG), 2013.

2 NATO HFM-234 (RTG). "Dictionary of Blast Injury Research Terms". Paris France: NATO HFM-234 (RTG), 2016.

3 NATO HFM-234 (RTG). "Guidelines for Using Animal Models in Blast Injury Research". Paris France: NATO HFM-234 (RTG), 2016. 